\title{
Randomized Approximation of Sobolev Embeddings
}

\author{
Stefan Heinrich \\ Department of Computer Science, University of Kaiserslautern, D-67653 \\ Kaiserslautern, Germany, heinrich@informatik.uni-kl.de
}

Summary. We study approximation of functions belonging to Sobolev spaces $W_{p}^{r}(Q)$ by randomized algorithms based on function values. Here $1 \leq p \leq \infty$, $Q=[0,1]^{d}$, and $r, d \in \mathbf{N}$. The error is measured in $L_{q}(Q)$, with $1 \leq q<\infty$, and we assume $r / d>1 / p-1 / q$, guaranteeing that $W_{p}^{r}(Q)$ is embedded into $L_{q}(Q)$. The optimal order of convergence for the case that $W_{p}^{r}(Q)$ is embedded even into $C(Q)$ is well-known. It is $n^{-r / d+\max (1 / p-1 / q, 0)}$ ( $n$ the number of function evaluations). This rate is already reached by deterministic algorithms, and randomization gives no speedup.

In this paper we are concerned with the case that $W_{p}^{r}(Q)$ is not embedded into $C(Q)$ (but, of course, still into $L_{q}(Q)$ ). For this situation approximation based on function values was not studied before. We prove that for randomized algorithms the above rate also holds, while for deterministic algorithms no rate whatsoever is possible. Thus, in the case of low smoothness, Monte Carlo approximation algorithms reach a considerable speedup over deterministic ones (up to $n^{-1+\varepsilon}$ for any $\varepsilon>0$ ).

We also give some applications to integration of functions and to approximation of solutions of elliptic PDE.

\section{Introduction}

Denote $\mathbf{N}=\{1,2, \ldots\}, \mathbf{N}_{0}=\{0,1,2, \ldots\}$, let $d \in \mathbf{N}$, and let $Q=[0,1]^{d}$ be the $d$-dimensional unit cube. For $1 \leq p \leq \infty$, let $L_{p}(Q)$ be the space of real-valued $p$-integrable functions, endowed with the norm

$$
\|f\|_{L_{p}(Q)}=\left(\int_{Q}|f(x)|^{p} d x\right)^{1 / p}
$$

if $p<\infty$, and

$$
\|f\|_{L_{\infty}(Q)}=\operatorname{ess}_{\sup _{x \in Q}}|f(x)| .
$$

For $r \in \mathbf{N}$ the Sobolev space $W_{p}^{r}(Q)$ consists of all functions $f \in L_{p}(Q)$ such that for all $\alpha=\left(\alpha_{1}, \ldots, \alpha_{d}\right) \in \mathbf{N}_{0}^{d}$ with $|\alpha|:=\sum_{j=1}^{d} \alpha_{j} \leq r$, the generalized partial derivative $D^{\alpha} f$ belongs to $L_{p}(Q)$. The norm on $W_{p}^{r}(Q)$ is defined as 


$$
\|f\|_{W_{p}^{r}(Q)}=\left(\sum_{|\alpha| \leq r}\left\|D^{\alpha} f\right\|_{L_{p}(Q)}^{p}\right)^{1 / p}
$$

if $p<\infty$, and

$$
\|f\|_{W_{\infty}^{r}(Q)}=\max _{|\alpha| \leq r}\left\|D^{\alpha} f\right\|_{L_{\infty}(Q)}
$$

Let $C(Q)$ denote the space of continuous functions on $Q$, equipped with the supremum norm. Let $1 \leq q<\infty$. By the Sobolev embedding theorem (see [Ada75], [Tri78]), for

$$
r / d>1 / p-1 / q
$$

$W_{p}^{r}(Q) \subset L_{q}(Q)$, and there is a constant $c>0$ such that for each $f \in W_{p}^{r}(Q)$

$$
\|f\|_{L_{q}(Q)} \leq c\|f\|_{W_{p}^{r}(Q)}
$$

Consequently, the embedding operator $J_{p q}: W_{p}^{r}(Q) \rightarrow L_{q}(Q)$ defined by $J_{p q} f=f$ is bounded. We shall study optimal approximation of $J_{p q}$ by randomized algorithms which use $n$ function values.

For $n \in \mathbf{N}$ we consider the class $\mathcal{A}_{n}^{\text {ran }}$ of randomized algorithms which are of the form $A=\left(A_{\omega}\right)_{\omega \in \Omega}$, where

$$
A_{\omega}(f)=\varphi_{\omega}\left(f\left(x_{1, \omega}\right), \ldots, f\left(x_{n, \omega}\right)\right),
$$

$(\Omega, \Sigma, \mathbf{P})$ is a probability space, for each $\omega \in \Omega, x_{i, \omega}$ is an element of $Q$ and $\varphi_{\omega}$ is a mapping from $\mathbf{R}^{n}$ to $L_{q}(Q)$, with the property that for each $f \in W_{p}^{r}(Q)$, the mapping

$$
\omega \in \Omega \rightarrow A_{\omega}(f)=\varphi_{\omega}\left(f\left(x_{1, \omega}\right), \ldots, f\left(x_{n, \omega}\right)\right)
$$

is a random variable with values in $L_{q}(Q)$ (that is, $\Sigma$-to-Borel measurable). Since elements of $W_{p}^{r}(Q)$ are equivalence classes of functions, relation (3) needs more explanation when $W_{p}^{r}(Q)$ is not embedded into $C(Q)$, and hence function values are, in general, not defined for such classes. We impose a further condition on the elements of $\mathcal{A}_{n}^{\text {ran }}$, let us call it consistency of the algorithm. We assume that whenever $f_{1}$ and $f_{2}$ are representatives of the same class $f \in W_{p}^{r}(Q)$, then

$$
\varphi_{\omega}\left(f_{1}\left(x_{1, \omega}\right), \ldots, f_{1}\left(x_{n, \omega}\right)\right)=\varphi_{\omega}\left(f_{2}\left(x_{1, \omega}\right), \ldots, f_{2}\left(x_{n, \omega}\right)\right) \quad \mathbf{P}-\text { a.s. }
$$

This means that $A_{\omega}\left(f_{1}\right)$ and $A_{\omega}\left(f_{2}\right)$ coincide almost surely, and in this sense we can take (3) as the definition of the random variable $A_{\omega}(f)$. A sufficient condition for consistency is obviously the following: For all $i$, the mapping $\omega \rightarrow x_{i, \omega}$ is Lebesgue measurable and for each subset $Q_{0} \subset Q$ of Lebesgue measure zero we have

$$
\mathbf{P}\left\{\omega \in \Omega: x_{i, \omega} \in Q_{0}\right\}=0,
$$


or equivalently, the distribution of the $x_{i, \omega}$ is absolutely continuous with respect to the Lebesgue measure.

The class $\mathcal{A}_{n}^{\text {ran }}$ contains the subclass of randomized linear algorithms they are of the form above with linear $\varphi_{\omega}$, thus

$$
A_{\omega}(f)=\sum_{i=1}^{n} f\left(x_{i, \omega}\right) \psi_{i, \omega},
$$

for certain $\psi_{i, \omega} \in L_{q}(Q)$.

Given any $1 \leq s<\infty$, the error of an algorithm $A \in \mathcal{A}_{n}^{\text {ran }}$ is defined as

$$
e^{(s)}\left(J_{p q}, A, B_{W_{p}^{r}(Q)}\right)=\sup _{f \in B_{W_{p}^{r}(Q)}}\left(\mathbf{E}\left\|f-A_{\omega}(f)\right\|_{L_{q}(Q)}^{s}\right)^{1 / s},
$$

where $\mathbf{E}$ is the expectation with respect to $\mathbf{P}$ and $B_{W_{p}^{r}(Q)}$ denotes the unit ball of $W_{p}^{r}(Q)$. The randomized $n$-th minimal error is defined as

$$
e_{n}^{\mathrm{ran}}\left(J_{p q}, B_{W_{p}^{r}(Q)}\right)=\inf _{A \in \mathcal{A}_{n}} e^{(1)}\left(J_{p q}, A, B_{W_{p}^{r}(Q)}\right) .
$$

Hence, no randomized algorithm that uses at most $n$ function values can provide a smaller error than $e_{n}^{\mathrm{ran}}\left(J_{p q}, B_{W_{p}^{r}(Q)}\right)$. We have chosen the $s=1$ case for the minimal error, which is convenient for the sequel. Statements for other $s$ can be read from the proofs below.

In terms of information-based complexity theory [TWW88], [Nov88], we consider randomized nonadaptive algorithms using standard information. In terms of [NT06], the $e_{n}^{\text {ran }}$ can be viewed as randomized sampling numbers.

In the case that $W_{p}^{r}(Q)$ is embedded into $C(Q)$, the order of $e_{n}^{\text {ran }}$ is wellknown:

$$
e_{n}^{\operatorname{ran}}\left(J_{p q}, B_{W_{p}^{r}(Q)}\right) \asymp n^{-r / d+\max (1 / p-1 / q, 0)},
$$

where we used the following notation: for sequences $\left(a_{n}\right)$ and $\left(b_{n}\right)$ of nonnegative reals we write $a_{n} \asymp b_{n}$ if there are constants $c_{1}, c_{2}>0$ and an $n_{0} \in \mathbf{N}$ such that $c_{1} a_{n} \leq b_{n} \leq c_{2} a_{n}$ for all $n \geq n_{0}$. Furthermore note that we often use the same symbols $c, c_{1}, \ldots$ for possibly different constants, also in sequences of relations.

The upper bound of (6) can be reached by deterministic methods, for example by piecewise polynomial approximation (see, e.g. [Cia78]). The lower bounds were shown in [Was89] $(p=q=\infty)$, [Nov88] $(1 \leq p \leq \infty, q=\infty)$, and [Mat91] $(1 \leq p, q \leq \infty)$. Recall from [Ada75] that $W_{p}^{r}(Q)$ is embedded into $C(Q)$ iff

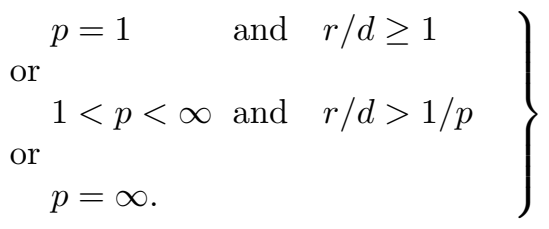


In this paper we are concerned with the case that (7) does not hold. Together with (1), this means we assume

$$
\left.\begin{array}{ll} 
& 1 \leq p, q<\infty \\
\text { and } & \\
1-1 / q<r / d<1 & \text { if } \quad p=1 \\
1 / p-1 / q<r / d \leq 1 / p & \text { if } \quad 1<p<\infty .
\end{array}\right\}
$$

Note that we demanded from the beginning that $W_{p}^{r}(Q)$ is embedded into $L_{q}(Q)$ (otherwise the operator $J_{p q}$ would not even be defined). So here we study the case that $W_{p}^{r}(Q)$ is embedded into $L_{q}(Q)$ but not into $C(Q)$. In this case approximation based on function values was not studied before. In the deterministic case there is a certain reason for this: function values are not defined any longer! However, this can easily be overcome. Namely, let us consider approximation on $B_{W_{p}^{r}(Q)} \cap C(Q)$, a dense subset of $B_{W_{p}^{r}(Q)}$. Now function values are defined, and the question arises which rate can be reached on the basis of Sobolev smoothness. It turns out that in the deterministic setting no rate whatsoever is possible on $B_{W_{p}^{r}(Q)} \cap C(Q)$. We discuss this issue in section 4 .

However, also the analysis of the randomized setting was restricted to the case (7) of embedding into $C(Q)$. In section 2 we present a randomized algorithm which reaches the rate (6) also in the case (8) of non-embedding into $C(Q)$. Hence, in contrast to the situation of embedding (7), randomized algorithms turn out to be superior to deterministic ones. We comment on this in more detail in section 4 .

Some applications to randomized integration of functions from $W_{p}^{r}(Q)$ and to approximation of solution operators of elliptic partial differential equations are given in Section 3.

\section{Randomized Approximation}

The following is the main result of this paper. We state it for $1 \leq p \leq \infty$, $1 \leq q<\infty$, since the proof works for all these cases, and thus shows that the method proposed also partly recovers the upper bound from (6) (having in mind though that the new part is the case in which (8) holds).

Theorem 1. Let $r, d \in \mathbf{N}, 1 \leq p \leq \infty, 1 \leq q<\infty$, and assume $r / d>$ $1 / p-1 / q$. Then

$$
e_{n}^{\operatorname{ran}}\left(J_{p q}, B_{W_{p}^{r}(Q)}\right) \asymp n^{-r / d+\max (1 / p-1 / q, 0)} .
$$

We proceed as follows. Under the assumptions of Theorem 1, we develop a general scheme for randomized approximation of functions. Its convergence is then analysed in Proposition 1. Based on this, the proof of Theorem 1 is given at the end of this section. 
Let $(\Omega, \Sigma, \mathbf{P})$ be a probability space, and for each $\omega \in \Omega$ let $P_{\omega}$ be any operator from $C(Q)$ to $L_{q}(Q)$ of the form

$$
P_{\omega} f=\sum_{j=1}^{\kappa} f\left(x_{j, \omega}\right) \psi_{j, \omega} \quad(f \in C(Q))
$$

with $x_{j, \omega} \in Q$ and $\psi_{j, \omega} \in L_{q}(Q)$. We assume that the mappings

$$
\omega \rightarrow x_{j, \omega}, \quad \omega \rightarrow \psi_{j, \omega}
$$

are random variables, the distributions of the $x_{j}$ being absolutely continuous with respect to the Lebesgue measure, that

$$
\begin{aligned}
& \left(\mathbf{E}\left|f\left(x_{j, \omega}\right)\right|^{q}\right)^{1 / q} \leq c\|f\|_{W_{p}^{r}(Q)} \quad\left(f \in W_{p}^{r}(Q)\right), \\
& \operatorname{ess} \sup _{\omega \in \Omega}\left\|\psi_{j, \omega}\right\|_{L_{q}(Q)} \leq c
\end{aligned}
$$

for $j=1, \ldots, \kappa$, and that for all $g \in \mathcal{P}_{r-1}(Q)$, the space of polynomials on $Q$ of degree not exceeding $r-1$,

$$
P_{\omega} g=g \quad \mathbf{P}-\text { a.s. }
$$

Families with such properties are easily constructed. For example, fix $0<$ $\delta<1$ and let

$$
P^{(1)} f=\sum_{j=1}^{\kappa} f\left(z_{j}\right) \psi_{j}
$$

be for $d=1$ the Lagrange interpolation operator of appropriate degree and for $d>1$ its tensor product, with $\left(z_{j}\right)_{j=1}^{\kappa}$ the uniform grid on $[0,1-\delta]^{d}$, and $\left(\psi_{j}\right)_{j=1}^{\kappa}$ the respective Lagrange polynomials, considered as functions on $\mathbf{R}^{d}$. Put $\Omega_{1}=[0, \delta]^{d}, \Sigma_{1}$ the $\sigma$-algebra of Lebesgue measurable sets and $\mathbf{P}_{1}$ the normalized on $[0, \delta]^{d}$ Lebesgue measure. For $\omega_{1} \in \Omega_{1}=[0, \delta]^{d}$ and $f \in C(Q)$ put

$$
\begin{gathered}
x_{j, \omega_{1}}=z_{j}+\omega_{1}, \\
\psi_{j, \omega_{1}}(x)=\psi_{j}\left(x-\omega_{1}\right) \quad(x \in Q),
\end{gathered}
$$

and

$$
\left(P_{\omega_{1}}^{(1)} f\right)(x)=\sum_{j=1}^{\kappa} f\left(z_{j}+\omega_{1}\right) \psi_{j}\left(x-\omega_{1}\right)
$$

Then we have for $f \in W_{p}^{r}(Q)$

$$
\begin{aligned}
\left(\mathbf{E}\left|f\left(x_{j, \omega_{1}}\right)\right|^{q}\right)^{1 / q} & =\left(\delta^{-d} \int_{[0, \delta]^{d}}\left|f\left(z_{j}+y\right)\right|^{q} d y\right)^{1 / q} \\
& \leq \delta^{-d / q}\|f\|_{L_{q}(Q)} \leq c \delta^{-d / q}\|f\|_{W_{p}^{r}(Q)}
\end{aligned}
$$


which shows that (10) is satisfied. It is readily checked that conditions (11) and (12) hold, as well.

Let $l \in \mathbf{N}_{0}$ and let

$$
Q=\bigcup_{i=1}^{2^{d l}} Q_{i}
$$

be the partition of $Q$ into $2^{d l}$ cubes of sidelength $2^{-l}$ and of disjoint interior. Let $x_{i}$ denote the point in $Q_{i}$ with minimal coordinates. Define the operators $E_{i}$ and $R_{i}$ on $L_{q}(Q)$ by setting for $f \in L_{q}(Q)$ and $x \in Q$

$$
\left(E_{i} f\right)(x)=f\left(x_{i}+2^{-l} x\right)
$$

and

$$
\left(R_{i} f\right)(x)=\chi_{Q_{i}}(x) f\left(2^{l}\left(x-x_{i}\right)\right)= \begin{cases}f\left(2^{l}\left(x-x_{i}\right)\right) & \text { if } x \in Q_{i} \\ 0 & \text { otherwise. }\end{cases}
$$

For $\omega \in \Omega$ set

$$
P_{l, \omega} f=\sum_{i=1}^{2^{d l}} R_{i} P_{\omega} E_{i} f=\sum_{i=1}^{2^{d l}} \sum_{j=1}^{\kappa} f\left(x_{i}+2^{-l} x_{j, \omega}\right) R_{i} \psi_{j, \omega}
$$

(observe that we use the same random variables $x_{j, \omega}$ for all $i$ ). It easily follows from the assumptions on $\left(P_{\omega}\right)_{\omega \in \Omega}$ that $\left(P_{l, \omega}\right)_{\omega \in \Omega}$ is an algorithm from $\mathcal{A}_{m}^{\text {ran }}$, where $m=\kappa 2^{d l}$. In fact it is a linear algorithm.

Proposition 1. Let $r, d \in \mathbf{N}, 1 \leq p \leq \infty, 1 \leq q<\infty$, and assume $r / d>1 / p-1 / q$. Let $\left(P_{\omega}\right)_{\omega \in \Omega}$ be as above satisfying (10), (11), (12), and let $\left(P_{l, \omega}\right)_{\omega \in \Omega}$ for $l \in \mathbf{N}_{0}$ be given by (17). Then there is a constant $c>0$ such that for all $l \in \mathbf{N}_{0}$ and $f \in W_{p}^{r}(Q)$

$$
\left(\mathbf{E}\left\|f-P_{l, \omega} f\right\|_{L_{q}(Q)}^{q}\right)^{1 / q} \leq c 2^{-r l+\max (1 / p-1 / q, 0) d l}\|f\|_{W_{p}^{r}(Q)} .
$$

Proof. It follows from (10) and (11) that for $f \in W_{p}^{r}(Q)$

$$
\begin{aligned}
\left(\mathbf{E}\left\|P_{\omega} f\right\|_{L_{q}(Q)}^{q}\right)^{1 / q} & \leq\left(\mathbf{E}\left(\sum_{j=1}^{\kappa}\left|f\left(x_{j, \omega}\right)\right|\left\|\psi_{j, \omega}\right\|_{L_{q}(Q)}\right)^{q}\right)^{1 / q} \\
& \leq c \sum_{j=1}^{\kappa}\left(\mathbf{E}\left|f\left(x_{j, \omega}\right)\right|^{q}\right)^{1 / q} \leq c\|f\|_{W_{p}^{r}(Q)} .
\end{aligned}
$$

We denote

$$
|f|_{r, p, Q}=\left(\sum_{|\alpha|=r}\left\|D^{\alpha} f\right\|_{L_{p}(Q)}^{p}\right)^{1 / p}
$$


if $p<\infty$ and

$$
|f|_{r, \infty, Q}=\max _{|\alpha|=r}\left\|D^{\alpha} f\right\|_{L_{\infty}(Q)} .
$$

Next we apply Theorem 3.1.1 from [Cia78]: there is a constant $c>0$ such that for all $f \in W_{p}^{r}(Q)$

$$
\inf _{g \in \mathcal{P}_{r-1}(Q)}\|f-g\|_{W_{p}^{r}(Q)} \leq c|f|_{r, p, Q} .
$$

It follows from (2), (12), (19), and (20) that

$$
\begin{aligned}
\left(\mathbf{E}\left\|f-P_{\omega} f\right\|_{L_{q}(Q)}^{q}\right)^{1 / q} & =\inf _{g \in \mathcal{P}_{r-1}(Q)}\left(\mathbf{E}\left\|(f-g)-P_{\omega}(f-g)\right\|_{L_{q}(Q)}^{q}\right)^{1 / q} \\
& \leq c \inf _{g \in \mathcal{P}_{r-1}(Q)}\|f-g\|_{W_{p}^{r}(Q)} \leq c|f|_{r, p, Q} .
\end{aligned}
$$

Clearly,

$$
\left\|R_{i} f\right\|_{L_{q}(Q)}=2^{-d l / q}\|f\|_{L_{q}(Q)} \quad\left(f \in L_{q}(Q)\right) .
$$

From (21) and (22) we obtain for all $f \in W_{p}^{r}(Q)$,

$$
\begin{aligned}
\left(\mathbf{E}\left\|f-P_{l, \omega} f\right\|_{L_{q}(Q)}^{q}\right)^{1 / q} & =\left(\mathbf{E}\left\|\sum_{i=1}^{2^{d l}}\left(R_{i} E_{i} f-R_{i} P_{\omega} E_{i} f\right)\right\|_{L_{q}(Q)}^{q}\right)^{1 / q} \\
& =\left(\mathbf{E} \sum_{i=1}^{2^{d l}}\left\|R_{i}\left(E_{i} f-P_{\omega} E_{i} f\right)\right\|_{L_{q}(Q)}^{q}\right)^{1 / q} \\
& =\left(2^{-d l} \sum_{i=1}^{2^{d l}} \mathbf{E}\left\|E_{i} f-P_{\omega} E_{i} f\right\|_{L_{q}(Q)}^{q}\right)^{1 / q} \\
& \leq c\left(2^{-d l} \sum_{i=1}^{2^{d l}}\left|E_{i} f\right|_{r, p, Q}^{q}\right)^{1 / q} \\
& \leq c 2^{\max (1 / p-1 / q, 0) d l}\left(2^{-d l} \sum_{i=1}^{2^{d l}}\left|E_{i} f\right|_{r, p, Q}^{p}\right)^{1 / p}
\end{aligned}
$$

and furthermore,

$$
\begin{aligned}
\left(2^{-d l} \sum_{i=1}^{2^{d l}}\left|E_{i} f\right|_{r, p, Q}^{p}\right)^{1 / p} & =\left(2^{-d l} \sum_{i=1}^{2^{d l}} \sum_{|\alpha|=r} \int_{Q}\left|D^{\alpha} f\left(x_{i}+2^{-l} x\right)\right|^{p} d x\right)^{1 / p} \\
& =2^{-r l}\left(\sum_{i=1}^{2^{d l}} \sum_{|\alpha|=r} \int_{Q_{i}}\left|D^{\alpha} f(y)\right|^{p} d y\right)^{1 / p} \\
& =2^{-r l}|f|_{r, p, Q} \leq 2^{-r l}\|f\|_{W_{p}^{r}(Q)} .
\end{aligned}
$$


(with the usual modifications for $p=\infty$ ). Combining the last two inequalities gives

$$
\left(\mathbf{E}\left\|f-P_{l, \omega} f\right\|_{L_{q}(Q)}^{q}\right)^{1 / q} \leq c 2^{-r l+\max (1 / p-1 / q, 0) d l}\|f\|_{W_{p}^{r}(Q)},
$$

which concludes the proof.

Proof of Theorem 1. Let $n \in \mathbf{N}$ and put

$$
l=\left\lceil\frac{\log _{2} n}{d}\right\rceil .
$$

Then $\left(P_{l, \omega}\right)_{\omega \in \Omega}$ belongs to $\mathcal{A}_{m}^{\text {ran }}$ with $m=\kappa 2^{d l} \leq c n$, so Proposition 1 together with (23) gives the upper bound in (9). The lower bound follows from standard techniques of information-based complexity (reduction to the average case on subsets formed by smooth bump functions) and is identical to that given in [Mat91] (see also [Nov88], [Hei93]). We omit it here.

\section{Some Applications}

First we consider integration. Let $Q=[0,1]^{d}$ and let $I: W_{p}^{r}(Q) \rightarrow \mathbf{R}$ be the integration operator

$$
\text { If }=\int_{Q} f(x) d x .
$$

Corollary 1. Let $r, d \in \mathbf{N}, 1 \leq p<\infty$, and put $\bar{p}=\min (p, 2)$. Then

$$
e_{n}^{\mathrm{ran}}\left(I, B_{W_{p}^{r}(Q)}\right) \asymp n^{-r / d-1+1 / \bar{p}} .
$$

This result was shown by Novak for $p \geq 2$ and for $p<2$ and $r / d \geq$ $1 / p-1 / 2$, that is, for the case that $W_{p}^{r}(Q)$ is embedded into $L_{2}(Q)$, see [Nov88], 2.2.9, and also the references therein for previous work. Our analysis supplies the remaining cases and a new technique: Novak used a result of [EZ60] on stochastic quadratures. For spaces embedded into $C(Q)$, another proof was given in [Hei93] using deterministic approximation as variance reduction (separation of the main part). What we present below might be viewed as a stochastic analogon of the latter.

Proof of Corollary 1. Fix $0<\delta<1$ and let $\left(\Omega_{1}, \Sigma_{1}, \mathbf{P}_{1}\right)$ be as defined above, following relation (13). Let $n \in \mathbf{N}$, let $l$ be given by (23), and let $P_{l, \omega_{1}}^{(1)}$ for $\omega_{1} \in \Omega_{1}$ be as defined in (13-17), that is, for $f \in W_{p}^{r}(Q)$ and $x \in Q$,

$$
\left(P_{l, \omega_{1}}^{(1)} f\right)(x)=\sum_{i=1}^{2^{d l}} \sum_{j=1}^{\kappa} f\left(x_{i}+2^{-l}\left(z_{j}+\omega_{1}\right)\right) \chi_{Q_{i}}(x) \psi_{j}\left(2^{l}\left(x-x_{i}\right)-\omega_{1}\right) .
$$


Finally, let $y_{k, \omega_{2}}(k=1, \ldots, n)$ be independent, uniformly distributed on $Q$ random variables over some probability space $\left(\Omega_{2}, \Sigma_{2}, \mathbf{P}_{2}\right)$. Define the algorithm $\left(A_{\omega_{2}}^{(2)}\right)_{\omega_{2} \in \Omega_{2}}$ to be the usual Monte Carlo method

$$
A_{\omega_{2}}^{(2)}(g)=\frac{1}{n} \sum_{k=1}^{n} g\left(y_{k, \omega_{2}}\right) .
$$

It is known that for $g \in L_{p}(Q)$

$$
\left(\mathbf{E}_{\omega_{2}}\left|I g-A_{\omega_{2}}^{(2)}(g)\right|^{\bar{p}}\right)^{1 / \bar{p}} \leq 2^{2 / \bar{p}-1} n^{-1+1 / \bar{p}}\|g\|_{L_{p}(Q)}
$$

(see [Hei93] for the case $1 \leq p<2$ ). Now we put

$$
(\Omega, \Sigma, \mathbf{P})=\left(\Omega_{1}, \Sigma_{1}, \mathbf{P}_{1}\right) \times\left(\Omega_{2}, \Sigma_{2}, \mathbf{P}_{2}\right)
$$

and define an algorithm $A=\left(A_{\omega}\right)_{\omega \in \Omega}$ by setting for $\omega=\left(\omega_{1}, \omega_{2}\right)$ and $f \in$ $W_{p}^{r}(Q)$

$$
A_{\omega}(f)=I P_{l, \omega_{1}}^{(1)} f+A_{\omega_{2}}^{(2)}\left(f-P_{l, \omega_{1}}^{(1)} f\right) .
$$

On the basis of (24) and (25) measurability and consistency readily follow, and we have $A \in \mathcal{A}_{m}^{\text {ran }}$ for $m=\kappa 2^{d l}+n \leq c n$. Moreover,

$$
I f-A_{\omega}(f)=I\left(f-P_{l, \omega_{1}}^{(1)} f\right)-A_{\omega_{2}}^{(2)}\left(f-P_{l, \omega_{1}}^{(1)} f\right) .
$$

Using Fubini's theorem, (26), and Proposition 1 for $q=p$, we derive

$$
\begin{aligned}
& \left(\mathbf{E}\left|I f-A_{\omega}(f)\right|^{\bar{p}}\right)^{1 / \bar{p}} \\
& =\left(\mathbf{E}_{\omega_{1}} \mathbf{E}_{\omega_{2}}\left|I\left(f-P_{l, \omega_{1}}^{(1)} f\right)-A_{\omega_{2}}^{(2)}\left(f-P_{l, \omega_{1}}^{(1)} f\right)\right|^{\bar{p}}\right)^{1 / \bar{p}} \\
& \leq c n^{-1+1 / \bar{p}}\left(\mathbf{E}_{\omega_{1}}\left\|f-P_{l, \omega_{1}}^{(1)} f\right\|_{L_{p}(Q)}^{\bar{p}}\right)^{1 / \bar{p}} \\
& \leq c n^{-1+1 / \bar{p}}\left(\mathbf{E}_{\omega_{1}}\left\|f-P_{l, \omega_{1}}^{(1)} f\right\|_{L_{p}(Q)}^{p}\right)^{1 / p} \\
& \leq c n^{-1+1 / \bar{p}-r / d}\|f\|_{W_{p}^{r}(Q)},
\end{aligned}
$$

concluding the proof of the upper bound. The lower bound is already contained in [Nov88], 2.2.9, Proposition 1.

Now we turn to elliptic problems. First results on the randomized information complexity of elliptic partial differential equations were obtained in [Hei06b]. The results above have some direct consequences for certain instances of this problem. Let $d, m \in \mathbf{N}, d \geq 2$, let $Q_{1} \subset \mathbf{R}^{d}$ be a bounded $C^{\infty}$ domain (see, e.g., [Tri78] for the definition), and let $\mathcal{L}$ be an elliptic differential operator of order $2 m$ on $Q_{1}$, that is 


$$
\mathcal{L} u=\sum_{|\alpha| \leq 2 m} a_{\alpha}(x) D^{\alpha} u(x),
$$

with boundary operators

$$
\mathcal{B}_{j} u=\sum_{|\alpha| \leq m_{j}} b_{j \alpha}(x) D^{\alpha} u(x)
$$

where $j=1, \ldots, m, m_{j} \leq 2 m-1$ and $a_{\alpha} \in C^{\infty}\left(Q_{1}\right)$ and $b_{j \alpha} \in C^{\infty}\left(\partial Q_{1}\right)$ are complex-valued infinitely differentiable functions. Consider the homogeneous boundary value problem

$$
\begin{aligned}
\mathcal{L} u(x) & =f(x) \quad\left(x \in Q_{1}^{0}\right) \\
\mathcal{B}_{j} u(x) & =0 \quad\left(x \in \partial Q_{1}\right) .
\end{aligned}
$$

We asssume that $\left(\mathcal{L},\left\{B_{j}\right\}\right)$ is regularly elliptic (see [Tri78], 5.2.1/4, for the definition), and that 0 is not in the spectrum of $\mathcal{L}$, considered as an unbounded operator in $L_{q}\left(Q_{1}\right)$ with domain of definition $W_{q,\left\{\mathcal{B}_{j}\right\}}^{2 m}\left(Q_{1}\right)$, where the latter denotes the subspace of $W_{q}^{2 m}\left(Q_{1}\right)$ consisting of those $f$ which satisfy (30). This implies that $\mathcal{L}$ is an isomorphism from $W_{q,\left\{\mathcal{B}_{j}\right\}}^{2 m}\left(Q_{1}\right)$ to $L_{q}\left(Q_{1}\right)$ for $1<q<\infty$, see [Tri78], Theorem 5.5.1(b). Now we put $S=\mathcal{L}^{-1} J_{p q}$, considered as an operator into $W_{q}^{2 m}\left(Q_{1}\right)$, that is,

$$
S: W_{p}^{r}\left(Q_{1}\right) \stackrel{J_{p q}}{\longrightarrow} L_{q}\left(Q_{1}\right) \stackrel{\mathcal{L}^{-1}}{\longrightarrow} W_{q}^{2 m}\left(Q_{1}\right) .
$$

Hence $S$ is the solution operator for the elliptic problem (29-30), where we consider the problem of approximating the full solution $u$, the right-hand side $f$ is supposed to belong to $W_{p}^{r}\left(Q_{1}\right)$, and the error is measured in the norm of $W_{q}^{2 m}\left(Q_{1}\right)$.

Corollary 2. Let $r, d \in \mathbf{N}, 1 \leq p \leq \infty, 1<q<\infty$, and $r / d>1 / p-1 / q$. Then

$$
e_{n}^{\operatorname{ran}}\left(S, B_{W_{p}^{r}\left(Q_{1}\right)}\right) \asymp n^{-r / d+\max (1 / p-1 / q, 0)} .
$$

Proof. Using local charts (like, e.g., in [Hei06b]) it is easy to extend Theorem 1 to smooth domains $Q_{1}$ in place of $Q=[0,1]^{d}$. It is also clear that the case of complex-valued functions is a direct consequence of the real case. From this and the above-mentioned fact that $\mathcal{L}^{-1}$ is an isomorphic embedding of $L_{q}\left(Q_{1}\right)$ into $W_{q}^{2 m}\left(Q_{1}\right)$ the upper bound follows.

So does the lower bound if we verify that algorithms with values in $\mathcal{L}^{-1}\left(L_{q}\left(Q_{1}\right)\right)=W_{q,\left\{\mathcal{B}_{j}\right\}}^{2 m}\left(Q_{1}\right)$, a subspace of $W_{q}^{2 m}\left(Q_{1}\right)$ containing $S\left(W_{p}^{r}\left(Q_{1}\right)\right)$, cannot be better (up to a constant) than algorithms with values in $W_{q}^{2 m}\left(Q_{1}\right)$. This, however, follows, e.g., from the fact that $W_{q,\left\{\mathcal{B}_{j}\right\}}^{2 m}\left(Q_{1}\right)$ is complemented in $W_{q}^{2 m}\left(Q_{1}\right)$, see [Tri78], Theorem 5.5.2(b), completing the proof. 
A similar approach (in the sense of using isomorphism properties to reduce approximation of solution operators to approximation of embeddings) was presented in [DNS06a, DNS06b] for the deterministic setting, with $q=2$. There, however, more general classes of operators and, besides function values, also arbitrary linear functionals are considered.

\section{Deterministic Approximation}

We already mentioned that for those $r, d$ and $p$ for which $W_{p}^{r}(Q)$ is embedded into $C(Q)$, the order of the randomized $n$-th minimal error coincides with that of the deterministic one. If the embedding does not hold, that is, if (8) is satisfied, the situation is different. First of all, since function values are not well-defined, we replace $B_{W_{p}^{r}(Q)}$ by the (dense) subset $B_{W_{p}^{r}(Q)} \cap C(Q)$. In this section we show that, although now function values are defined, the Sobolev smoothness $W_{p}^{r}(Q)$ does not lead to any rate at all for the deterministic setting. That is, the deterministic $n$-th minimal error is bounded from below by a positive constant.

Put $F=B_{W_{p}^{r}(Q)} \cap C(Q)$, let $n \in \mathbf{N}$ and let $\mathcal{A}_{n}^{\text {det }}$ be the class of all deterministic algorithms for the approximation of $J_{p q}$ on $F$, which are of the form

$$
A(f)=\varphi\left(f\left(x_{1}\right), \ldots, f\left(x_{n}\right)\right),
$$

where $x_{i} \in Q(i=1, \ldots, n)$ and $\varphi: \mathbf{R}^{n} \rightarrow L_{q}(Q)$ is an arbitrary mapping. The error on $F$ is defined as

$$
e\left(J_{p q}, A, F\right)=\sup _{f \in F}\|S(f)-A(f)\|_{L_{q}(Q)} .
$$

The deterministic $n$-th minimal error is defined as

$$
e_{n}^{\operatorname{det}}\left(J_{p q}, F\right)=\inf _{A \in \mathcal{A}_{n}^{\text {det }}} e\left(J_{p q}, A, F\right) .
$$

Proposition 2. Let $1 \leq p, q<\infty$ and $r / d>1 / p-1 / q$. Assume that either

$$
\frac{r}{d}<\frac{1}{p}
$$

or

$$
\frac{r}{d}=\frac{1}{p} \quad \text { and } \quad 1<p<\infty
$$

Then

$$
e_{n}^{\operatorname{det}}\left(J_{p q}, B_{W_{p}^{r}(Q)} \cap C(Q)\right) \asymp 1 .
$$

For the proof we need the following lemma. Let $B(0, \varrho)$ denote the closed ball in $\mathbf{R}^{d}$ of radius $\varrho$ around 0 . 
Lemma 1. Assume that (33) or (34) holds. Then there is a sequence of functions

$$
\left(f_{m}\right)_{m=1}^{\infty} \subset W_{p}^{r}\left(\mathbf{R}^{d}\right) \cap C^{\infty}\left(\mathbf{R}^{d}\right)
$$

such that for all $m$

$$
f_{m}(0)=1, \quad \operatorname{supp} f_{m} \subseteq B\left(0, \frac{1}{m}\right),
$$

and

$$
\lim _{m \rightarrow \infty}\left\|f_{m}\right\|_{W_{p}^{r}\left(\mathbf{R}^{d}\right)}=0 .
$$

Proof. In case of (34) this is a combination of well-known facts from function space theory. Let $\psi \in C^{\infty}\left(\mathbf{R}^{d}\right)$ be such that $\psi \geq 0$,

$$
\psi(x)=\left\{\begin{array}{lll}
1 & \text { if } & |x| \leq \frac{1}{2} \\
0 & \text { if } & |x| \geq 1
\end{array}\right.
$$

and

$$
\int_{\mathbf{R}^{d}} \psi(x) d x=1
$$

Put

$$
g(x)=\left\{\begin{array}{lll}
\psi(x) \ln \ln \frac{3}{|x|} & \text { if } & 0<|x|<1 \\
0 & \text { if } & |x| \geq 1
\end{array}\right.
$$

Then

$$
\|g\|_{W_{p}^{r}\left(\mathbf{R}^{d}\right)}<\infty, \quad \operatorname{supp} g \subseteq B(0,1), \quad \lim _{x \rightarrow 0} g(x)=+\infty,
$$

see [Ada75], Example 5.26. Furthermore, setting

$$
h_{m}=\psi_{m} * g \quad \text { with } \quad \psi_{m}(x)=m^{d} \psi(m x) \quad\left(x \in \mathbf{R}^{d}, m \in \mathbf{N}\right),
$$

we get, using Lemma 3.15 of [Ada75] and (38),

$$
\begin{aligned}
& h_{m} \in C^{\infty}\left(\mathbf{R}^{d}\right), \quad \operatorname{supp} h_{m} \subseteq B(0,2), \quad h_{m}(0)>0 \quad(m \in \mathbf{N}), \\
& \sup _{m \in \mathbf{N}}\left\|h_{m}\right\|_{W_{p}^{r}\left(\mathbf{R}^{d}\right)}<\infty, \quad \lim _{m \rightarrow \infty} h_{m}(0)=+\infty .
\end{aligned}
$$

Finally we define $f_{m}$ by

$$
f_{m}(x)=h_{m}(0)^{-1} h_{m}(2 m x) \quad\left(x \in \mathbf{R}^{d}, m \in \mathbf{N}\right) .
$$

Relation (34) implies

$$
\left\|f_{m}\right\|_{W_{p}^{r}\left(\mathbf{R}^{d}\right)} \leq h_{m}(0)^{-1}\left\|h_{m}(2 m \cdot)\right\|_{W_{p}^{r}\left(\mathbf{R}^{d}\right)} \leq c h_{m}(0)^{-1} \rightarrow 0,
$$

while (36) is obviously fulfilled by definition. This completes the proof in the case of (34). 
If (33) holds, we choose $\psi$ as above and put

$$
f_{m}(x)=\psi(m x) \quad\left(x \in \mathbf{R}^{d}, m \in \mathbf{N}\right) .
$$

Clearly, (36) is satisfied, and it follows from (33) that

$$
\left\|f_{m}\right\|_{W_{p}^{r}\left(\mathbf{R}^{d}\right)} \leq c m^{r-d / p} \rightarrow 0 \quad(m \rightarrow \infty) .
$$

Proof of Proposition 2. The upper bound follows from the fact that $J_{p q}$ is bounded. To prove the lower bound, let $x_{1}, \ldots, x_{n}$ be any fixed distinct points in $Q$. For $m \in \mathbf{N}$ consider the function $v_{m} \in C(Q)$ given by

$$
v_{m}(x)=\left(1+n\left\|f_{m}\right\|_{W_{p}^{r}\left(\mathbf{R}^{d}\right)}\right)^{-1}\left(1-\sum_{i=1}^{n} f_{m}\left(x-x_{i}\right)\right) \quad(x \in Q) .
$$

We have

$$
\left\|v_{m}\right\|_{W_{p}^{r}(Q)} \leq 1
$$

and, using (37),

$$
\begin{aligned}
\left\|v_{m}\right\|_{L_{q}(Q)} & \geq \int_{Q} v_{m}(x) d x \\
& =\left(1+n\left\|f_{m}\right\|_{W_{p}^{r}\left(\mathbf{R}^{d}\right)}\right)^{-1}\left(1-\sum_{i=1}^{n} \int_{Q} f_{m}\left(x-x_{i}\right) d x\right) \\
& \geq\left(1+n\left\|f_{m}\right\|_{W_{p}^{r}\left(\mathbf{R}^{d}\right)}\right)^{-1}\left(1-n\left\|f_{m}\right\|_{W_{p}^{r}\left(\mathbf{R}^{d}\right)}\right) \rightarrow 1
\end{aligned}
$$

as $m \rightarrow \infty$. Finally, by (36), for $m$ large enough,

$$
v_{m}\left(x_{i}\right)=0 \quad(i=1, \ldots, n) .
$$

Now (39-41) combined with standard results from information-based complexity theory [TWW88], Ch. 3.1, prove Proposition 2.

Proposition 2 was independently obtained by Novak and Woźniakowski (unpublished notes).

\section{Comments}

Comparing the rates in Theorem 1 and in Proposition 2 for the case (8) of non-embedding into $C(Q)$, we see that on $B_{W_{p}^{r}(Q)} \cap C(Q)$ randomization gives a speedup over the deterministic setting of

$$
\frac{e_{n}^{\mathrm{ran}}\left(J_{p q}, B_{W_{p}^{r}(Q)} \cap C(Q)\right)}{e_{n}^{\operatorname{det}}\left(J_{p q}, B_{W_{p}^{r}(Q)} \cap C(Q)\right)} \asymp n^{-r / d+\max (1 / p-1 / q, 0)},
$$


which is non-trivial in all cases, since $r / d>\max (1 / p-1 / q, 0)$ by assumption. If $p=1$, the maximal exponent of the speedup is $r / d$, reached for $q=1$, and $r / d$ can be arbitrarily close to 1 . If $1<p<\infty$, the maximal exponent of the speedup is again $r / d$ and can now be as large as $1 / p$, reached for $q \leq p$.

Let us also mention that the lower bounds presented here for the randomized setting hold true for the more general case of adaptive (standard) information, as introduced e.g. in [Hei06a]. Similarly for the deterministic case. The latter follows from general results [TWW88].

As is readily seen from its proof, Proposition 2 remains true if $J_{p q}$ is replaced by $I$ of Corollary 1 , the parameter $q$ and the condition $r / d>1 / p-1 / q$ being omitted. Furthermore, the argument used in the proof of Corollary 2 shows that we can also replace $J_{p q}$ in Proposition 2 by $S$.

\section{References}

[Ada75] Adams, R.A.: Sobolev Spaces. Academic Press, New York (1975)

[Cia78] Ciarlet, P.G.: The Finite Element Method for Elliptic Problems. NorthHolland, Amsterdam (1978)

[DNS06a] Dahlke, S., Novak, E., Sickel,W.: Optimal approximation of elliptic problems by linear and nonlinear mappings I, J. Complexity 22, 29-49 (2006)

[DNS06b] Dahlke, S., Novak, E., Sickel,W.: Optimal approximation of elliptic problems by linear and nonlinear mappings II, J. Complexity 22, 549-603 (2006)

[EZ60] Ermakov, S.M., Zolotukhin, V.G.: Polynomial approximation and the Monte Carlo method, Theor. Prob. Appl. 5, 428-431 (1960)

[Hei93] Heinrich, S.: Random approximation in numerical analysis. In: Bierstedt, K.D., Pietsch, A., Ruess, W.M., Vogt, D. (eds) Functional Analysis. Marcel Dekker, New York, 123-171 (1993)

[Hei06a] Heinrich, S.: Monte Carlo approximation of weakly singular integral operators. J. Complexity 22, 192-219 (2006)

[Hei06b] Heinrich, S.: The randomized information complexity of elliptic PDE. J. Complexity 22, 220-249 (2006)

[Mat91] Mathé, P.: Random approximation of Sobolev embeddings. J. Complexity 7, 261-281 (1991)

[Nov88] Novak, E.: Deterministic and Stochastic Error Bounds in Numerical Analysis. Lecture Notes in Mathematics 1349, Springer-Verlag, Berlin (1988)

[NT06] Novak, E., Triebel, H.: Function spaces in Lipschitz domains and optimal rates of convergence for sampling. Constr. Approx. 23, 325-350 (2006)

[TWW88] Traub, J.F., Wasilkowski, G.W., Woźniakowski, H.: Information-Based Complexity. Academic Press, New York (1988)

[Tri78] Triebel, H.: Interpolation Theory, Function Spaces, Differential Operators. North-Holland, Amsterdam, New York, Oxford (1978)

[Was89] Wasilkowski, G.W.: Randomization for continuous problems. J. Complexity 5, 195-218 (1989) 